\title{
Factors affecting the persistency of milk production in Jersey crossbred cattle
}

\begin{abstract}
Data on part lactation yields (50-days, 70-days, $1^{\text {st }} 100,2^{\text {nd }} 100$ days, $3^{\text {rd }} 100,182$ days) and 305-days milk yield comprising all lactations of 378 Jersey crossbred cattle, maintained at the Eastern Regional Station of ICAR-NDRI, Kalyani over a period of 35 years (19822016) were collected to study the effect of genetic and non-genetic factors on lactation persistency of cattle. Five lactation persistency indices of animals $\left(\mathrm{P}_{21}, \mathrm{P}_{31}, \mathrm{P}_{32}, \mathrm{P}_{4}\right.$ and $\mathrm{P}_{5}$ ) were calculated using ratio method. Least squares analysis of variance with fitting constant was applied to determine the effect of genetic and non-genetic factors on different persistency indices of Jersey crossbred cattle. In the present study, the least squares means of five different measures of lactation persistency viz., $\mathrm{P}_{21}, \mathrm{P}_{31}, \mathrm{P}_{32}, \mathrm{P}_{4}$ and $\mathrm{P}_{5}$ were $0.76 \pm 0.01,0.59 \pm 0.01,0.80 \pm 0.01,4.70 \pm 0.06$ and $1.30 \pm 0.02$ respectively. Random effect of sire showed significant $(\mathrm{P}<0.05)$ influence on some of the persistency indices (viz., $\mathrm{P}_{21}, \mathrm{P}_{4}$ and $\left.\mathrm{P}_{5}\right)$. Period of calving had significant $(\mathrm{P}<0.01)$ effect on all the five persistency indices. Season of calving showed significant $(\mathrm{P}<0.01)$ influence on $\mathrm{P}_{21}, \mathrm{P}_{31}, \mathrm{P}_{4}$ and $\mathrm{P}_{5}$. Cows that calved during rainy season showed the highest persistency in milk yield. Parity of animals had significant effect on all measures of persistency indices and cows in first parity showed the highest persistency as compared to cows of later parities. Genetic groups of animals did not show significant $(\mathrm{P}>0.05)$ effect on any of the persistency indices considered for this study.
\end{abstract}

Keywords: lactation persistency, environmental factors, crossbred cattle
Volume 7 Issue 6 - 2018

\section{Subrata Koloi, Kanai Pathak, Rajalaxmi Behera, Mandal DK, Karunakaran M, Dutta TK, Ajoy Mandal}

Eastern Regional Station of ICAR-National Dairy Research Institute, Kalyani -741235 , India

Correspondence: Ajoy Mandal, Eastern Regional Station of ICAR-National Dairy Research Institute, Kalyani - 74I235, India, Email ajoymendal@gmail.com

Received: August 13,2018 | Published: December II, 2018

\section{Introduction}

Lactation persistency, an economically important trait of dairy animal, can be defined as the ability of a cow to continue milk production at a high level after the peak yield. ${ }^{1}$ Cows that sustain high rate of milk secretion for longer period in lactation are known as persistent producers while other goes dry or drop the yield within a few weeks and hence called as non-persistent. High lactation persistency is associated with slow rate of decline in production, whereas low lactation persistency is associated with a rapid rate of decline. In any dairy industry, animals producing milk at a high level of persistency are considered as economical as it will lead to reduction and stabilization of production cost and ultimately yield more profit. The persistency of lactation is affected by various environmental factors such as genetic group, sire effect, herd management, lactation number, feeding, gestation and season of calving of animals. Therefore, the present study was undertaken to determine the effect of genetic and non-genetic factors on lactation persistency of crossbred cattle.

\section{Material and methods}

The present study was conducted on Jersey crossbred cows to identify the genetic and non-genetic sources of variations in persistency indices of milk production. Data on part lactation yields (50-days, 70days, $1^{\text {st }} 100,2^{\text {nd }} 100$ days, $3^{\text {rd }} 100,182$ days), 305 -days milk yield comprising all lactations of 378 Jersey crossbred cattle, maintained at the Eastern Regional Station of ICAR-NDRI, Kalyani over a period of 35 years (1982-2016) divided into 7 periods were collected and used. Each year was divided into 3 seasons viz. winter (NovemberFebruary), summer (March-June) and rainy (July-October). Animals were divided into 10 genetic groups depending upon their level of exotic inheritance. Five lactation persistency indices of animals $\left(\mathrm{P}_{21}\right.$, $\mathrm{P}_{31}, \mathrm{P}_{32}, \mathrm{P}_{4}$ and $\mathrm{P}_{5}$ ) were calculated using ratio method as follows:

I. $\mathrm{P}_{21}$ is the persistency index No. $1=2^{\text {nd }} 100$ days milk yield $/ 1$ st 100 days milk yield,

II. $\mathrm{P}_{31}$ is the persistency index No. $2=3^{\text {rd }} 100$ days milk yield $/ 1$ st 100 days milk yield,

III. $\mathrm{P}_{32}$ is the persistency index No. $3=3^{\text {rd }} 100$ days milk yield $/ 2^{\text {nd }} 100$ days milk yield,

IV. $\mathrm{P}_{4}$ is the persistency index No. $4=305$ days milk yield/50 days milk yield and

V. $\mathrm{P}_{5}$ is the persistency index No. $5=(182$ days milk yield -70 days milk yield)/70 days milk yield. since, the data were distributed unequally and disproportionately among sub-classed, so, data were analyzed by using the LSML (least squares maximum likelihood) computer programme.

The following mixed model was used:

$Y_{i j k l m n}=\mu+B_{i}+P_{j}+(P a)_{k}+S_{l}+(G G)_{m}+e_{i j k l m n}$

Where,

$\mathrm{Y}_{\mathrm{ijklmn}}=\mathrm{n}^{\text {th }}$ observation on lactation persistency of crossbred cow calved during $\mathrm{j}^{\text {th }}$ period, $\mathrm{k}^{\text {th }}$ parity, $\mathrm{l}^{\text {th }}$ season and belonging to $\mathrm{m}^{\text {th }}$ genetic group.

$\mu \quad=$ Overall mean

$\mathrm{B}_{\mathrm{i}} \quad=$ Random effect of $\mathrm{i}^{\text {th }}$ sire

$\mathrm{P}_{\mathrm{j}} \quad=$ Fixed effects of $\mathrm{j}^{\text {th }}$ period of calving $(\mathrm{k}=1,2,3 \ldots \ldots 7)$

$(\mathrm{Pa})_{\mathrm{k}}=$ Fixed effects of $\mathrm{k}^{\text {th }}$ parity of animal $(\mathrm{l}=1,2,3 \ldots \ldots 7)$

$\mathrm{S}_{1}=$ Fixed effects of $1^{\text {th }}$ season of calving $(j=1,2,3)$

$(\mathrm{GG})_{\mathrm{m}}=$ Effect of $\mathrm{m}^{\text {th }}$ genetic group $(\mathrm{m}=1,2,3 \ldots \ldots 10)$

$\mathrm{e}_{\mathrm{jklmn}}=$ Random error $\sim \operatorname{NID}\left(0, \sigma_{\mathrm{e}}^{2}\right)$ 


\section{Results and discussion}

The overall least squares means of $\mathrm{P}_{21}, \mathrm{P}_{31}, \mathrm{P}_{32}, \mathrm{P}_{4}$ and $\mathrm{P}_{5}$ were $0.76 \pm 0.01, \quad 0.59 \pm 0.01, \quad 0.80 \pm 0.01, \quad 4.70 \pm 0.06$ and $1.30 \pm 0.02$ respectively. Random effect of sire showed significant $(\mathrm{P}<0.05)$ influence on $\mathrm{P}_{21}, \mathrm{P}_{4}$ and $\mathrm{P}_{5}$. Period of calving had significant $(\mathrm{P}<0.05)$ effect on all the five indices of lactation persistency. Of Jersey crossbred cows (Table 1). Similar significant effect of period of calving on lactation persistency of cattle was observed by Kumar et al., ${ }^{2}$ in Karan Fries cattle, Garudkar et al., ${ }^{3}$ in Phule Triveni cattle and Sharma et al., ${ }^{4}$ in HF cattle. In this study, there was no definite trend for lactation persistency over the periods but animals calved during 1992-1996 and 2002-2006 had comparatively higher persistency whereas, the cows that calved in 2007-2011 and 2012-2016 had lower persistent than others. Moreover, all the persistency indices of animals exhibited constant trend in the later periods, which may be ascribed as same managemental practices in the herd during the periods. The parity of animal had significant $(\mathrm{P}<0.05)$ effect on all the persistency indices of milk production (Table 1). The highest lactation persistency was observed in Jersey crossbred cows of first parity in the present study. Similar significant effect of parity of cow on lactation persistency was observed by various workers ${ }^{5-8}$ The reason of cows of first parity were more persistent in this study may be explained as presence of more number of secretary cells in mammary gland, which maintain their secretary activity for a longer duration in first lactation as compared to subsequent lactations where although the amount of milk secretion by each secretary cell is increased but it fails to maintain their secretary activity for a longer duration and finally get sloughed off at early stage of lactation. Season of calving also showed significant $(\mathrm{P}<0.05)$ effect on $\mathrm{P}_{21}, \mathrm{P}_{31}, \mathrm{P}_{4}$ and $\mathrm{P}_{5}$ persistency measures. Similar significant effects of season of calving on lactation persistency was observed by Kumar et al., ${ }^{2}$ Yilmaz et al., ${ }^{8}$ \& Guler et al., ${ }^{9}$ in different breeds of cattle. In the present study, rainy calves were more persistent than summer and winter calves. The reason may be due to sufficient availability of green fodder during rainy season which is followed by a comfortable temperature of ensuing winter season post peak yield. Cows calved during summer season were least persistent which may be due to increased temperature with high humidity. These findings were in agreement with that of Kumar et al., ${ }^{2}$ who reported highest persistency in rainy calves in Karan-Fries cattle. In the present study, genetic group of animals did not show significant effect on persistency indices of milk production. Although cows with $50 \%$ Jersey inheritance showed more persistency as compared to cows with less than or more than $50 \%$ Jersey inheritance. Sharma et al. ${ }^{4}$ also observed non-significant effect of genetic group of animals on persistency indices of milk production in HF crossbred cattle.

Table I Least-squares means along with standard errors of persistency indices of milk production in relation to period of calving, parity, season of calving and genetic group in Jersey crossbred cattle

\begin{tabular}{|c|c|c|c|c|c|}
\hline Effects & P2I & P3I & P32 & P4 & P5 \\
\hline Overall Mean & $\begin{array}{l}0.76 \pm 0.01 \\
(1291)\end{array}$ & $\begin{array}{l}0.59 \pm 0.01 \\
(977)\end{array}$ & $\begin{array}{l}0.80 \pm 0.01 \\
(985)\end{array}$ & $\begin{array}{l}4.70 \pm 0.06 \\
(980)\end{array}$ & $\begin{array}{l}1.30 \pm 0.02 \\
(13 \mid I)\end{array}$ \\
\hline \multicolumn{6}{|c|}{ Period of Calving } \\
\hline PdI (1982-86) & $0.73^{\mathrm{abc}} \pm 0.05$ & $\begin{array}{l}0.60^{\mathrm{bc}} \pm 0.05 \\
(41)\end{array}$ & $\begin{array}{l}0.98^{\mathrm{a}} \pm 0.07 \\
(43)\end{array}$ & $\left.4.7\right|^{\mathrm{abc}} \pm 0.27(43)$ & $1.25^{\mathrm{abc}} \pm 0.08(5 \mathrm{I})$ \\
\hline Pd2 (|987-9|) & $\begin{array}{l}0.80^{\mathrm{ab}} \pm 0.03 \\
(133)\end{array}$ & $\begin{array}{l}0.62^{2 \mathrm{~b}} \pm 0.04 \\
(105)\end{array}$ & $0.86^{\mathrm{ab}} \pm 0.05(106)$ & $\begin{array}{l}4.92^{2 \mathrm{~b}} \pm 0.19 \\
(104)\end{array}$ & $\begin{array}{l}1.38^{\mathrm{ab}} \pm 0.06 \\
(138)\end{array}$ \\
\hline Pd3 (1992-96) & $\begin{array}{l}0.80^{\mathrm{ab}} \pm 0.02 \\
(185)\end{array}$ & $\begin{array}{l}0.6 \mathrm{I}^{\mathrm{ab}} \pm 0.02 \\
(15 \mathrm{I})\end{array}$ & $\begin{array}{l}0.79^{b c} \pm 0.03 \\
(152)\end{array}$ & $\begin{array}{l}5.04^{a} \pm 0.13 \\
(150)\end{array}$ & $1.40^{\mathrm{a}} \pm 0.04(184)$ \\
\hline Pd4 (1997-0I) & $\begin{array}{l}0.76^{\mathrm{abc}} \pm 0.02 \\
(227)\end{array}$ & $\begin{array}{l}0.56^{\mathrm{abc}} \pm 0.02 \\
(186)\end{array}$ & $\begin{array}{l}0.76^{b c} \pm 0.03 \\
(187)\end{array}$ & $\begin{array}{l}4.60^{\mathrm{bc}} \pm 0.11 \\
(185)\end{array}$ & $\begin{array}{l}1.3 \mathrm{I}^{\mathrm{ab}} \pm 0.03 \\
(230)\end{array}$ \\
\hline Pd5 (2002-06) & $0.8 \mathrm{I}^{\mathrm{a}} \pm 0.02(194)$ & $0.63^{\mathrm{a}} \pm 0.03(143)$ & $\begin{array}{l}0.79^{b c} \pm 0.03 \\
(145)\end{array}$ & $\begin{array}{l}\left.4.9\right|^{\mathrm{ab}} \pm 0.13 \\
(146)\end{array}$ & $\begin{array}{l}1.35^{\mathrm{ab}} \pm 0.04 \\
(197)\end{array}$ \\
\hline Pd6 (2007-I I) & $\begin{array}{l}0.72^{\mathrm{bc}} \pm 0.03 \\
(24 I)\end{array}$ & $\begin{array}{l}0.52^{c} \pm 0.03 \\
(173)\end{array}$ & $\begin{array}{l}0.70^{c} \pm 0.04 \\
(174)\end{array}$ & $\begin{array}{l}4.45^{\text {bd }} \pm 0.17 \\
(174)\end{array}$ & $\begin{array}{l}1.23^{b c} \pm 0.05 \\
(247)\end{array}$ \\
\hline Pd7 (2012-16) & $\begin{array}{l}0.69^{c} \pm 0.04 \\
(295)\end{array}$ & $\begin{array}{l}0.57^{\mathrm{abc}} \pm 0.05 \\
(178)\end{array}$ & $\begin{array}{l}\left.0.7\right|^{\mathrm{bc}} \pm 0.06 \\
(178)\end{array}$ & $\begin{array}{l}4.27^{c} \pm 0.22 \\
(178)\end{array}$ & $\begin{array}{l}1.16^{c} \pm 0.07 \\
(264)\end{array}$ \\
\hline \multicolumn{6}{|c|}{ Parity of animal } \\
\hline PI & $\begin{array}{l}0.80^{\mathrm{a}} \pm 0.01 \\
(374)\end{array}$ & $\begin{array}{l}0.67^{a} \pm 0.02 \\
(300)\end{array}$ & $\begin{array}{l}0.85^{\mathrm{a}} \pm 0.02 \\
(301)\end{array}$ & $\begin{array}{l}5.00^{\mathrm{a}} \pm 0.09 \\
(298)\end{array}$ & $\begin{array}{l}1.36^{\mathrm{a}} \pm 0.03 \\
(38 \mathrm{I})\end{array}$ \\
\hline P2 & $\begin{array}{l}0.76^{\mathrm{b}} \pm 0.01 \\
(295)\end{array}$ & $\begin{array}{l}0.59^{\mathrm{b}} \pm 0.01 \\
(209)\end{array}$ & $\begin{array}{l}0.80^{\mathrm{a}} \pm 0.02 \\
(215)\end{array}$ & $\begin{array}{l}4.64^{b} \pm 0.08 \\
(214)\end{array}$ & $\begin{array}{l}1.27^{\mathrm{b}} \pm 0.03 \\
(299)\end{array}$ \\
\hline P3 & $\begin{array}{l}0.76^{\mathrm{b}} \pm 0.01 \\
(220)\end{array}$ & $\begin{array}{l}0.58^{\mathrm{b}} \pm 0.01 \\
(164)\end{array}$ & $\begin{array}{l}0.78^{b} \pm 0.02 \\
(164)\end{array}$ & $\begin{array}{l}4.63^{b} \pm 0.09 \\
(165)\end{array}$ & $\begin{array}{l}1.29^{\mathrm{ab}} \pm 0.03 \\
(222)\end{array}$ \\
\hline P4 & $\begin{array}{l}0.75^{b} \pm 0.02 \\
(146)\end{array}$ & $\begin{array}{l}0.57^{b} \pm 0.02 \\
(110)\end{array}$ & $\begin{array}{l}0.82^{\mathrm{a}} \pm 0.02 \\
(\mathrm{II} 2)\end{array}$ & $\begin{array}{l}4.48^{b} \pm 0.09 \\
(109)\end{array}$ & $\begin{array}{l}1.29^{\mathrm{ab}} \pm 0.03 \\
(146)\end{array}$ \\
\hline P5 & $\begin{array}{l}0.76^{b} \pm 0.02 \\
(109)\end{array}$ & $\begin{array}{l}0.57^{b} \pm 0.02 \\
(86)\end{array}$ & $0.78^{\mathrm{ab}} \pm 0.03$ & $\begin{array}{l}4.72^{b} \pm 0.10 \\
(86)\end{array}$ & $\begin{array}{l}\mathrm{I} .3 \mathrm{I}^{\mathrm{ab}} \pm 0.03 \\
(\mathrm{I} \mathrm{I0})\end{array}$ \\
\hline
\end{tabular}




\begin{tabular}{|c|c|c|c|c|c|}
\hline Effects & P2I & P3I & P32 & P4 & P5 \\
\hline P6 & $\begin{array}{l}0.74^{b} \pm 0.02 \\
(69)\end{array}$ & $\begin{array}{l}0.57^{b} \pm 0.02 \\
(54)\end{array}$ & $\begin{array}{l}0.80^{\mathrm{a}} \pm 0.03 \\
(54)\end{array}$ & $\begin{array}{l}4.72^{\mathrm{ab}} \pm 0.13 \\
(55)\end{array}$ & $\begin{array}{l}1.28^{\mathrm{ab}} \pm 0.04 \\
(72)\end{array}$ \\
\hline P7 & $\begin{array}{l}0.75^{\mathrm{b}} \pm 0.02 \\
(78)\end{array}$ & $\begin{array}{l}0.56^{\mathrm{b}} \pm 0.03 \\
(54)\end{array}$ & $\begin{array}{l}0.76^{b} \pm 0.04 \\
(54)\end{array}$ & $\begin{array}{l}4.71 \pm 0.15 \\
(53)\end{array}$ & $\begin{array}{l}1.29^{\mathrm{ab}} \pm 0.04 \\
(8 \mathrm{I})\end{array}$ \\
\hline \multicolumn{6}{|l|}{ Season of calving } \\
\hline SI (Winter) & $\begin{array}{l}0.77^{\mathrm{a}} \pm 0.01 \\
(4 \mid 8)\end{array}$ & $\begin{array}{l}0.56^{\mathrm{b}} \pm 0.01 \\
(325)\end{array}$ & $\begin{array}{l}0.78 \pm 0.02 \\
(328)\end{array}$ & $\begin{array}{l}4.64^{b} \pm 0.07 \\
(327)\end{array}$ & $\begin{array}{l}1.32^{\mathrm{a}} \pm 0.02 \\
(422)\end{array}$ \\
\hline S2 (Summer) & $\begin{array}{l}0.72^{\mathrm{b}} \pm 0.01 \\
(440)\end{array}$ & $\begin{array}{l}0.57^{\mathrm{b}} \pm 0.0 \mathrm{I} \\
(34 \mathrm{I})\end{array}$ & $\begin{array}{l}0.8 I \pm 0.02 \\
(34 I)\end{array}$ & $\begin{array}{l}4.59^{b} \pm 0.07 \\
(336)\end{array}$ & $\begin{array}{l}1.25^{b} \pm 0.02 \\
(446)\end{array}$ \\
\hline S3 (Rainy) & $\begin{array}{l}0.79^{\mathrm{a}} \pm 0.01 \\
(433)\end{array}$ & $\begin{array}{l}0.64^{a} \pm 0.01 \\
(311)\end{array}$ & $\begin{array}{l}0.81 \pm 0.02 \\
(316)\end{array}$ & $\begin{array}{l}4.87^{\mathrm{a}} \pm 0.07 \\
(317)\end{array}$ & $\begin{array}{l}1.32^{\mathrm{a}} \pm 0.02 \\
(443)\end{array}$ \\
\hline \multicolumn{6}{|l|}{ Genetic group of animal } \\
\hline G-I $(1 / 2 J \times 1 / 2 R S)$ & $\begin{array}{l}0.78 \pm 0.02 \\
(99)\end{array}$ & $\begin{array}{l}0.60 \pm 0.02 \\
(81)\end{array}$ & $\begin{array}{l}0.80 \pm 0.03 \\
(81)\end{array}$ & $\begin{array}{l}4.77 \pm 0.11 \\
(81)\end{array}$ & $\begin{array}{l}1.32 \pm 0.04 \\
(99)\end{array}$ \\
\hline G-2 $(1 / 2 \mathrm{~J} \times 1 / 2 T)$ & $\begin{array}{l}0.73 \pm 0.02 \\
(243)\end{array}$ & $\begin{array}{l}0.59 \pm 0.03 \\
(178)\end{array}$ & $\begin{array}{l}0.83 \pm 0.04 \\
(183)\end{array}$ & $\begin{array}{l}4.73 \pm 0.14 \\
(184)\end{array}$ & $\begin{array}{l}1.26 \pm 0.05 \\
(245)\end{array}$ \\
\hline G-3 ( $\geq 50 \%$ to $62.5 \% \mathrm{~J})$ & $\begin{array}{l}0.77 \pm 0.02 \\
(229)\end{array}$ & $\begin{array}{l}0.57 \pm 0.02 \\
(172)\end{array}$ & $\begin{array}{l}0.79 \pm 0.02 \\
(173)\end{array}$ & $\begin{array}{l}4.69 \pm 0.10 \\
(171)\end{array}$ & $\begin{array}{l}1.35 \pm 0.03 \\
(230)\end{array}$ \\
\hline G-4 $(I / 2 \mathrm{~J} \times \mathrm{I} / 4 \mathrm{RS} \times \mathrm{I} / 4 \mathrm{~T})$ & $\begin{array}{l}0.79 \pm 0.02 \\
(192)\end{array}$ & $\begin{array}{l}0.62 \pm 0.02 \\
(147)\end{array}$ & $\begin{array}{l}0.79 \pm 0.03 \\
(146)\end{array}$ & $\begin{array}{l}4.87 \pm 0.11 \\
(145)\end{array}$ & $\begin{array}{l}1.35 \pm 0.03 \\
(195)\end{array}$ \\
\hline $\begin{array}{l}\text { G-5 }(1 / 2 \mathrm{I} \times 1 / 4 \mathrm{RS} \times 1 / 8 \mathrm{~T} \times \\
\mathrm{I} / 8 \mathrm{D})\end{array}$ & $\begin{array}{l}0.75 \pm 0.02 \\
(110)\end{array}$ & $\begin{array}{l}0.58 \pm 0.02 \\
(73)\end{array}$ & $\begin{array}{l}0.79 \pm 0.03 \\
(73)\end{array}$ & $\begin{array}{l}4.75 \pm 0.12 \\
(73)\end{array}$ & $\begin{array}{l}1.29 \pm 0.04 \\
(I 12)\end{array}$ \\
\hline G-6 $(1 / 2 J \times 3 / 8 R S \times 1 / 8 T)$ & $\begin{array}{l}0.78 \pm 0.02 \\
(\mathrm{III})\end{array}$ & $\begin{array}{l}0.59 \pm 0.02 \\
(80)\end{array}$ & $\begin{array}{l}0.78 \pm 0.03 \\
(79)\end{array}$ & $\begin{array}{l}4.89 \pm 0.13 \\
(79)\end{array}$ & $\begin{array}{l}1.36 \pm 0.04 \\
(114)\end{array}$ \\
\hline G-7 (Misc. 50\% J) & $0.79 \pm 0.03(75)$ & $\begin{array}{l}0.59 \pm 0.03 \\
(61)\end{array}$ & $\begin{array}{l}0.79 \pm 0.04 \\
(64)\end{array}$ & $\begin{array}{l}4.71 \pm 0.16 \\
(64)\end{array}$ & $\begin{array}{l}1.31 \pm 0.05 \\
(78)\end{array}$ \\
\hline$G-8(\leq 50 \% \mathrm{~J})$ & $\begin{array}{l}0.74 \pm 0.03 \\
(47)\end{array}$ & $\begin{array}{l}0.57 \pm 0.03 \\
(31)\end{array}$ & $\begin{array}{l}0.81 \pm 0.05 \\
(30)\end{array}$ & $\begin{array}{l}4.50 \pm 0.19 \\
(30)\end{array}$ & $\begin{array}{l}1.27 \pm 0.06 \\
(50)\end{array}$ \\
\hline G-9 (>62.5 to $75 \% \mathrm{~J})$ & $\begin{array}{l}0.76 \pm 0.03 \\
(98)\end{array}$ & $\begin{array}{l}0.58 \pm 0.03 \\
(8 I)\end{array}$ & $\begin{array}{l}0.80 \pm 0.04 \\
(83)\end{array}$ & $\begin{array}{l}4.64 \pm 0.14 \\
(8 I)\end{array}$ & $\begin{array}{l}1.26 \pm 0.05 \\
(100)\end{array}$ \\
\hline G-10 (>75\% J) & $\begin{array}{l}0.70 \pm 0.03 \\
(87)\end{array}$ & $\begin{array}{l}0.58 \pm 0.03 \\
(73)\end{array}$ & $\begin{array}{l}0.80 \pm 0.04 \\
(73)\end{array}$ & $\begin{array}{l}4.46 \pm 0.15 \\
(72)\end{array}$ & $\begin{array}{l}1.20 \pm 0.05 \\
(88)\end{array}$ \\
\hline
\end{tabular}

Figures in the parenthesis indicate number of observations Means with different superscripts in each subclass within a column differ significantly $(P<0.05)$ from each other.

\section{Conclusion}

Different persistency indices of milk production of Jersey crossbred cattle were significantly affected by various environmental factors, which indicate the necessity of improving the managemental conditions of the farm for better productivity.

\section{Acknowledgment}

The authors are thankful to Director, ICAR-NDRI, Karnal and Head, ERS of ICAR-NDRI, Kalyani, India for providing necessary facilities for conducting this work. The help rendered by staff of cattle yard for maintaining and providing the records for this study is also duly acknowledged.

\section{Conflicts of interest}

The author declares that there are no conflicts of interest.

\section{References}

1. Jamrozik J, Jensen G, Schaeffer LR, et al. Analysis of persistency of lactation calculated from a random regression test day model. Interbull Bulletin.1998;16:64-68.

2. Kumar A, Singh A. Genetic evaluation of persistency of milk production and its association with production and reproduction traits in Karan Fries cattle. Indian Journal of Dairy Science. 2006;59(1):19-24.

3. Garudkar SR, Pachpute ST, Deokar DK. Studies on Persistency of Milk Yield and Its Association with Production Traits in Phule Triveni Synthetic Cow. International Journal of Current Microbiology and Applied Science. 2018;6:1585-1589.

4. Sharma N, Narang R, Ratwan P, et al. Genetic analysis of persistency in $\mathrm{HF}$ crossbred cattle at an organized farm of northern India. Tropical Animal Health and Production. 2018;50(6):1219-1225.

5. Zurwan A, Moaeen-ud-Din M, Bilal G, et al. Estimation of genetic parameters for persistency of lactation in Sahiwal dairy cattle. Pakistan Journal of Zoology. 2017;49(3):877-882. 
6. Otwinowska-Mindur A, Ptak E. Genetic analysis of persistency in the Polish Holstein-Friesian cows. Animal Science Papers and Reports. 2015;33:109-117.

7. Jingar S, Mehla RK, Singh M, et al. Lactation Curve Pattern and Prediction of Milk Production Performance in Crossbred Cows. Journal of Veterinary Medicine. 2014.
8. Yilmaz H, Koc A. A research on milk yield, persistency, milk constituents and somatic cell count of Red Holstein cows raised under Mediterranean climatic conditions. Bulgarian Journal of Agricultural Science. 2013;19(6):1401-1407.

9. Guler O, Yanar M. Factors Influencing the Shape of Lactation Curve and Persistency of Holstein Friesian Cows in High Altitude of Eastern Turkey. Journal of Applied Animal Research. 2009;35(1):39-44. 